OPEN ACCESS

Edited by:

Randy Yeh,

Memorial Sloan Kettering Cancer

Center, United States

Reviewed by:

llja Ciernik,

Städtische Klinikum Dessau, Germany

*Correspondence:

Roger Sun

roger.sun@gustaveroussy.fr

Eric Deutsch

eric.deutsch@gustaveroussy.fr

Specialty section:

This article was submitted to

Cancer Imaging and

Image-directed Interventions,

a section of the journal

Frontiers in Oncology

Received: 27 April 2020 Accepted: 23 October 2020 Published: 18 November 2020

Citation:

Sun R, Ammari S, Bockel S, Achkar S, Merad M, Dercle L, Rivera S,

Chargari $C$ and Deutsch E (2020)

Optimization of Patient Management

During the COVID-19 Pandemic:

Chest CT Scan and PCR as

Gatekeepers of the Radiation

Therapy Workflow.

Front. Oncol. 10:556334.

doi: 10.3389/fonc.2020.556334

\section{Optimization of Patient Management During the COVID-19 Pandemic: Chest CT Scan and PCR as Gatekeepers of the Radiation Therapy Workflow}

\author{
Roger Sun ${ }^{1 *}$, Samy Ammari ${ }^{2}$, Sophie Bockel ${ }^{1}$, Samir Achkar ${ }^{1}$, Mansouria Merad ${ }^{3}$, \\ Laurent Dercle ${ }^{4}$, Sofia Rivera ${ }^{1}$, Cyrus Chargari ${ }^{1}$ and Eric Deutsch ${ }^{1 *}$ \\ 1 Gustave Roussy, Département de Radiothérapie, INSERM 1030, Université Paris-Saclay, Villejuif, France, 2 Gustave \\ Roussy, Département d'Imagerie Médicale, Université Paris-Saclay, Villejuif, France, ${ }^{3}$ Gustave Roussy, Département \\ d'Oncologie Médicale, Université Paris-Saclay, Villejuif, France, ${ }^{4}$ Department of Radiology, Columbia University Irving Medical \\ Center, New York, NY, United States
}

Keywords: coronavirus disease, radiotherapy, chest CT, workflow, PCR

\section{INTRODUCTION}

The coronavirus disease 2019 (COVID-19) has been rapidly spreading since the first patients were described in Wuhan, China in late December 2019 (1). As of mid-July 2020, nearly 15 million of confirmed cases and 600,000 deaths have been reported worldwide (2). This global pandemic and international health crisis has challenged healthcare providers to profoundly re-organize healthcare systems in order to ensure the continuity of essential treatments, while limiting the risk to patients and healthcare providers, and simultaneously handling shortages in personnel, beds, and equipment (3).

Increasing evidence suggests that cancer patients and especially those undergoing treatment might be at higher risk of developing severe forms of COVID-19 (4-7). Indeed, anticancer treatment such as chemotherapy and radiotherapy that induce neutropenia and/or lymphopenia, as well as targeted therapy or immune-check point inhibitors, may worsen the course of COVID-19, although this is still debated $(3,8)$ For this reason, cancer societies have recommended to adapt the management of cancer patients by de-escalating cytotoxic chemotherapy, delaying non-urgent treatments, and considering non-surgical options when feasible $(9,10)$. In radiation oncology, some indications remain non-deferrable, such as chemoradiotherapy for locally advanced tumors, brachytherapy, and urgent palliative treatment, particularly in patients with rapidly growing tumors (11). In addition, radiotherapy capacity may even need to be increased as an alternative treatment to compensate for surgery cancellations. While hypofractionated radiotherapy schedules may help to limit the number of hospital visits, daily sessions for at least 2 weeks remain the rule for most curative treatments, with some plans lasting up to $7-8$ weeks $(12,13)$. Moreover, strategy relying only on patient selection and prioritizing treatment management alone may not be sufficient in a prolonged healthcare crises (10). Active and effective strategies of early detection of COVID-19 
should therefore be discussed and tailored for radiation oncology departments, especially since a significant proportion of patients are asymptomatic (14).

\section{SARS-COV-2 DETECTION AND COVID-19 DIAGNOSIS}

COVID-19 is an infectious respiratory disease caused by a novel coronavirus called SARS-CoV-2 (15). The accepted standard routine laboratory method to diagnose COVID-19 consists of the use of reverse transcription polymerase chain reaction (rt-PCR) to detect viral RNA in respiratory samples. However, performance of nasopharyngeal and oropharyngeal swabs is questioned since several studies have shown a high number of false negative results $(16,17)$. In a cohort of 213 patients with mild to severe symptoms of COVID-19, Yang et al. reported positive rates ranging from $73.3 \%$ for nasal swabs performed less than 7 days after illness onset on patients with severe symptoms and $53.6 \%$ for nasal swabs performed more than 8 days after illness onset on patients with mild symptoms. Wang et al. reported low positive rates of $32 \%$ for pharyngeal swabs (126/ 398 patients) and $63 \%$ for nasal swabs (5/8 patients). Moreover, rt-PCR has other limitations including the availability of testing kits, the need for adapted infrastructure, training of personnel for high quality swab samples, and relative lengthy turnaround times for test results.

On the other hand, some authors have suggested computed tomography (CT) could be pivotal for the diagnosis and the screening of COVID-19 $(18,19)$. In a cohort of 1,014 patients, $\mathrm{Ai}$ et al. reported a positive rate of 59\% for rt-PCR (601/1014) and $88 \%$ for chest CT $(888 / 1014)$, with $97 \%$ of positive rt-PCR patients having also a positive chest CT (580/601) (18). The higher sensitivity of chest CT compared to rt-PCR was confirmed in a recent meta-analysis [94\% (5\% CI: 91\%, 96\%) vs. $89 \%$ (95\% CI: $81 \%, 94 \%)$, respectively] (20). However, this study highlighted the low specificity of chest CT [37\% (95\% CI: $26 \%, 50 \%)$ ] with poor positive predictive value (PPV) for populations with low expected prevalence of COVID-19, limiting its routine use for mass screening and diagnosis in the overall population, especially since CT scans also expose people to ionizing radiation. Therefore, the general consensus is to not recommend chest CT for diagnosing COVID-19 and to reserve CT for patients with worsening symptoms $(21,22)$. However, several authors have suggested that imaging could help patient triage in a resource-constrained environment, especially when the pre-test probability of COVID-19 is high $(21,22)$. Nevertheless, with a high negative predictive value (NPV) estimated between $90.6 \%$ and $99.8 \%$, chest CT imaging could still be very valuable in specific situations, such as in screening patients undergoing radiotherapy.

Recently, serological tests have become more widespread (23). Detection of IgM antibodies associated with symptoms is highly suggestive of SARS-CoV-2 infection. IgM antibodies may be detectable around 5 to 10 days after the onset of symptoms, with a mean time for seroconversion of 10 to 12 days for IgM, and 12 to 14 days for IgG (23-26). However, whether an antibody response with neutralizing antibodies is associated with protective immunity is still unclear, and higher IgG concentration have been reported in patients with severe COVID-19 infection (27). While these tests were not recommended for systematic screening by the World Health Organization (28), they were recommended by the French Haute Autorité de Santé in patients with negative RT-PCR and clinical suspicion for COVID-19 (29).

\section{OPTIMIZING MEDICAL IMAGING IN RADIOTHERAPY DURING THE COVID-19 PANDEMIC}

\section{Feasibility and Benefit of Chest CT in Radiation Therapy}

Radiation therapy requires each patient to undergo a simulation CT scan for treatment planning. This CT allows the visualization and delineation of target tumor volumes to irradiate and normal tissues to spare, the calculation and the optimization of the radiation dose, and the reproducibility of patient positioning (30). An additional chest CT scan or extending the scan coverage of a simulation CT could therefore be easily performed for each patient without modifying patient's visit or the radiotherapy workflow. Moreover, the expected additional delivered radiation dose to the chest is low, around $12 \mathrm{mGy}$ [interquartile range (IQR), 7-17 mGy] $(31,32)$, and may be considered as negligible for patients undergoing radiotherapy. Indeed, even for nonthoracic treatment, the estimated dose at $30 \mathrm{~cm}$ from the irradiated field is estimated to be $0.05 \%-0.7 \%$ of the delivered dose (33). For example, the estimated dose received to the chest for a pelvic treatment of 45 Gy would correspond to 2-26 times the dose of a chest CT. In addition, free-breathing chest CTs are routinely performed during the CT simulation for patients with lung and breast cancers, metastases to the thoracic and lumbar spine, and sometimes for head and neck cancers. In certain indications, such as in breast cancer, deep inspiration breath hold radiotherapy technique allows acquisition of breath-hold chest CT.

For patients with other cancers, the additional chest CT could also serve as a baseline for reference, in addition to screening for COVID-19. Indeed, cancer patients may have progressive cancer- or cancer-treatment related lung abnormalities, which may be complicated to interpret if the patient becomes symptomatic. Moreover, the rate of incidental findings in cancer patients may be relatively high: a study of 510 patients reported incidental findings in up to $28 \%$ of patients, including $3.4 \%$ of patients with a significant finding that either changed the cancer therapy or required immediate treatment (34).

\section{Early Detection of COVID-19 in Patients Undergoing Radiotherapy}

The lack of large epidemiological studies and the difficulty of estimating the true incidence of COVID-19 cases make it 
challenging to determine whether cancer patients are more or less vulnerable to SARS-CoV-2 in comparison with the overall population. Indeed, among COVID-19 patients, an important proportion of patients are asymptomatic, but may still be contagious (35-37). A voluntary screening on 10,797 patients in Iceland, with 87 COVID-19 positive patients $(0.8 \%)$ of whom $57 \%$ were asymptomatic (14). Moreover, with an incubation period between 3 to 6 days $(15,38)$, a study estimated that up to $44 \%$ [95\% CI (25-69)] of secondary cases may have been infected by pre-symptomatic cases (36). Cancer patients undergoing treatment may have compromised immunity, potentially increasing their morbidity and mortality from COVID-19. Miyashita et al. reported 5,688 COVID-19 patients, including 334 patients with cancer. Intubation was more frequent in cancer patients [RR: 1.89 95\% CI (1.37-2.61)], but there was no significant excess risk of death (5). Kuderer et al. reported in a study of 928 patients with history of cancer that there was an increased 30-day mortality in patients with active cancer [progressing vs. remission: Odds ratio $=5.20$, 95\% CI $(2.77-$ 9.77)] (39).

Radiotherapy treatment requires frequent hospital visits for the patients, a flow of multiple patients per day on the same machine, and involvement of an entire team of healthcare professionals including a medical dosimetrist, medical physicist, radiation oncologist, radiation therapists, oncology nurses, caregivers, and medical secretaries. Strategies for early detection of COVID-19 are therefore of utmost importance to ensure patient and medical staff safety during the COVID-19 pandemic, especially in regions with high prevalence and documented community spread.

\section{Proposal of COVID-19 Testing for Asymptomatic Patients in Radiation Oncology Departments}

A workflow including systematic nasopharyngeal swab and chest CT for asymptomatic patients is currently being evaluated in our Radiotherapy department, as the prevalence of COVID-19 was high in our region, with up to $11.9 \%$ of individuals infected (range: $7.6 \%$ to $19.4 \%$ ) according to epidemiologic models (40). Inspiratory breath hold chest acquisition is made during the simulation CT, with the same CT acquisition protocols conventionally used in radiology. While free-breathing acquisitions are usually done in radiotherapy, they are more susceptible to motion artifacts and may obscure or even mimic subtle ground-glass opacities commonly seen in COVID-19, and regular cone-beam CT (CBCT) positioning review may require comparison with reference chest CT. Images are then reviewed by both the treating radiation oncologist and an experienced radiologist for imaging findings suggestive of COVID-19 such as ground-glass opacity, condensation, reticulation, interlobular or intralobular septal thickening, nodules, and distribution of the lesions (41). For suspected COVID-19 patients on either radiological findings or clinical symptoms but negative RTPCR, directed nasopharyngeal swab and inflammatory blood test are repeated before the onset of radiotherapy. This procedure allows the classification of patients into three different categories with specific management according to the COVID-19 probability (Figure 1):

1) Confirmed or probable cases: We propose using a dedicated treatment room with adapted personal protective equipment \{protective masks against inhalation of droplets, such as FFP2 (filtering facepiece type 2) masks [standard NF EN 149 (42)], gown, gloves, eye protection, and apron $\}$ and a dedicated accelerator for the irradiation of suspected and/or confirmed COVID-19 patients, which may help to avoid deferring treatment. On a case by case basis taking into account symptoms, deferring radiotherapy after the acute phase of infection is discussed. However, this approach using a dedicated room for confirmed or probable cases is only feasible in large centers with a high number of accelerators.

2) Uncertain cases: We propose in these patients close monitoring of clinical symptoms and repeating nasopharyngeal swabs. Further investigation might be proposed according to the multidisciplinary team, such as CT follow-up or other microbiology investigation and specimens. These patients are preferably scheduled for treatment at the end of the day.

3) Unlikely cases: We propose using standard personal protective equipment and routine radiotherapy treatment protocol.

In addition to CT scan and rt-PCR screening, we have undertaken a global reorganization of our radiation oncology department with implementation of barrier precautions and social distancing. Prior to entry, patients are asked to answer a short checklist to screen for clinical symptoms, such as fevers, chills, cough, etc., and reception staff measure the temperature of patients at the entrance. All medical staff and patients are required to wear surgical masks in the department. The flow of patients in the radiation oncology department has also been rethought. The simulation CT is dedicated to COVID-19 patients only on Friday, allowing a two-day interval before using the scanner again for patients without COVID-19. Whenever possible, we encourage COVID-19 patients to wait for their session in their car or in the taxi in order to limit the time spent in the waiting room, and therefore, limiting the risk of SARS-CoV-2 transmission.

These screening strategies have the most potential and maximum benefit during the peak of COVID-19 cases and adjustments need to be made according to the evolution of the pandemic and the pre-test probability of COVID-19 in a particular area, as low prevalence of COVID-19 leads to a low PPV, especially for chest CT (20). For instance, while systematic baseline RT-PCR testing is recommended in Canada by the Ontario provincial Ministry of Health guidelines for cancer patients undergoing immunosuppressive cancer treatment, including radiation therapy, high priority testing criteria for asymptomatic patients in the event of testing limitations were defined, included age $\geq 60$ years, performance status $\geq 2$, comorbid conditions or impaired immunity, significant smoking history and lung tissue in the radiation treatment volume (43) 


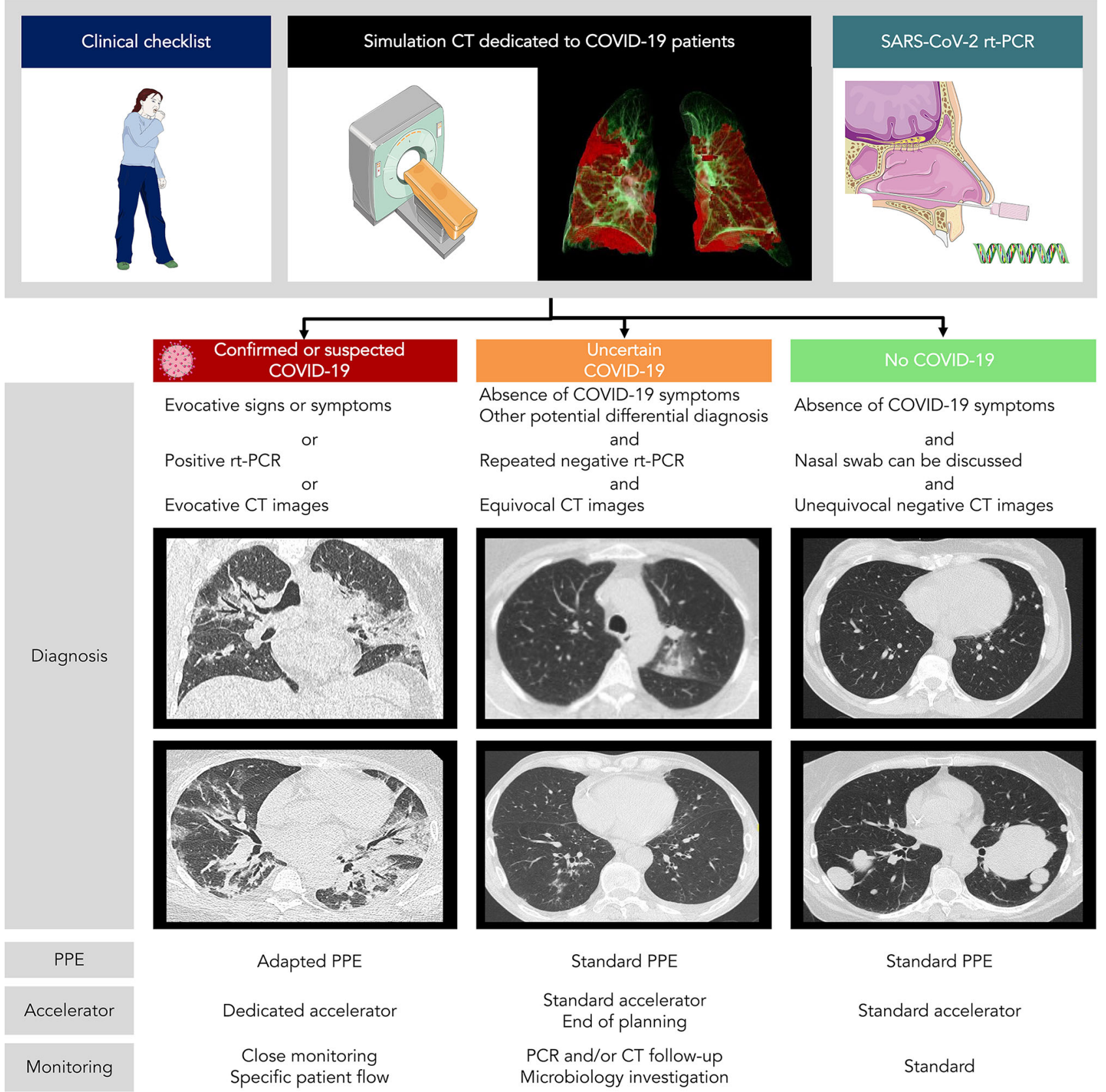

FIGURE 1 | COVID-19 patients management in radiotherapy according to chest CT + rt-PCR screening. PPE, personal protective equipment.

\section{CONCLUSION}

The COVID-19 pandemic has significantly impacted the delivery of care to cancer patients, leading to delays in providing adequate treatment. While postponing treatments and providing remote consultations were feasible in the early stages of the pandemic, these temporary measures are no longer sustainable as the pandemic continues. Hospitals and medical services need to develop and adopt long-term strategies to continue providing cancer care during the pandemic. In patients undergoing radiotherapy, we propose using chest CT and rt-PCR screening for early detection of COVID-19, especially since a number of patients are asymptomatic and cancer patients might be more vulnerable than the overall population. Rethinking the flow of patients is also critical to allow the continuation of care, with implementation of barrier precautions and social distancing. 


\section{AUTHOR CONTRIBUTIONS}

RS and ED prepared the first draft of the manuscript. LD conceived the figure. SB, SR, SAm, MM, SAc, LD, and CC reviewed the manuscript. All authors contributed to the article and approved the submitted version.

\section{REFERENCES}

1. Zhu N, Zhang D, Wang W, Li X, Yang B, Song J, et al. A Novel Coronavirus from Patients with Pneumonia in China, 2019. N Engl J Med (2020) 382 (8):727-33. doi: 10.1056/NEJMoa2001017

2. World Health Organization. Novel Coronavirus (2019- $n$ CoV) situation reports. Situation Report (2020). p. 183. Available at: https://www.who.int/ emergencies/diseases/novel-coronavirus-2019/situation-reports Last Accessed 21 July 2020.

3. van de Haar J, Hoes LR, Coles CE, Seamon K, Fröhling S, Jäger D, et al. Caring for patients with cancer in the COVID-19 era. Nat Med (2020) 26:1-7. 16 avr. doi: 10.1038/s41591-020-0948-7

4. Liang W, Guan W, Chen R, Wang W, Li J, Xu K, et al. Cancer patients in SARS-CoV-2 infection: a nationwide analysis in China. Lancet Oncol (2020) 21(3):335-7. doi: 10.1016/S1470-2045(20)30096-6

5. Miyashita H, Mikami T, Chopra N, Yamada T, Chernyavsky S, Rizk D, et al. Do Patients with Cancer Have a Poorer Prognosis of COVID-19? An Experience in New York City. Ann Oncol (2020) 31(8):1088-9. doi: 10.1016/ j.annonc.2020.04.006

6. Tian Y, Qiu X, Wang C, Zhao J, Jiang X, Niu W, et al. Cancer associates with risk and severe events of COVID-19: A systematic review and meta-analysis. Int J Cancer (2020). doi: 10.1002/ijc.33213

7. Li Q, Chen L, Li Q, He W, Yu J, Chen L, et al. Cancer increases risk of inhospital death from COVID-19 in persons <65 years and those not in complete remission. Leukemia (2020) 34(9):2384-91. doi: 10.1038/s41375020-0986-7

8. Lee LYW, Cazier JB, Starkey T, Turnbull CDUK Coronavirus Cancer Monitoring Project Team, , Kerr R, et al. COVID-19 mortality in patients with cancer on chemotherapy or other anticancer treatments: a prospective cohort study. Lancet Lond Engl (2020) 395(10241):1919-26. doi: 10.1016/ S0140-6736(20)31173-9

9. Burki TK. Cancer guidelines during the COVID-19 pandemic. Lancet Oncol (2020) 21(5):629-630. doi: 10.1016/S1470-2045(20)30217-5

10. Hanna TP, Evans GA, Booth CM. Cancer, COVID-19 and the precautionary principle: prioritizing treatment during a global pandemic. Nat Rev Clin Oncol (2020) 17(5):268-70. doi: 10.1038/s41571-020-0362-6

11. Chargari C, Chopra S, Viswanathan AN, Deutsch E. Brachytherapy Issues And Priorities In The Context of COVID-19 Outbreak. Adv Radiat Oncol (2020) 5(4):640-3. doi: 10.1016/j.adro.2020.04.034

12. Zaorsky NG, Yu JB, McBride SM, Dess RT, Jackson WC, Mahal BA, et al. Prostate Cancer Radiotherapy Recommendations in Response to COVID-19. Adv Radiat Oncol (2020) 5(4):659-65. doi: 10.1016/j.adro.2020.03.010

13. Thomson DJ, Palma D, Guckenberger M, Balermpas P, Beitler JJ, Blanchard P, et al. Practice recommendations for risk-adapted head and neck cancer radiotherapy during the COVID-19 pandemic: an ASTRO-ESTRO consensus statement. Int J Radiat Oncol Biol Phys (2020) 107(4):618-27. doi: 10.1016/j.radonc.2020.04.019

14. Gudbjartsson DF, Helgason A, Jonsson H, Magnusson OT, Melsted P, Norddahl GL, et al. Spread of SARS-CoV-2 in the Icelandic Population. N Engl J Med (2020) 382(24):2302-15. doi: 10.1101/2020. 03.26.20044446

15. Li Q, Guan X, Wu P, Wang X, Zhou L, Tong Y, et al. Early Transmission Dynamics in Wuhan, China, of Novel Coronavirus-Infected Pneumonia. N Engl J Med (2020) 382(13):1199-207. doi: 10.1056/NEJMoa2001316

16. Wang W, Xu Y, Gao R, Lu R, Han K, Wu G, et al. Detection of SARS-CoV-2 in Different Types of Clinical Specimens. JAMA (2020) 323(18):1843-4. doi: 10.1001/jama.2020.3786

17. Yang Y, Yang M, Shen C, Wang F, Yuan J, Li J, et al. Evaluating the accuracy of different respiratory specimens in the laboratory diagnosis and monitoring the

\section{ACKNOWLEDGMENTS}

We thank Gianfranco Brusadin, Simon Corbin, Frederik Hubert, Virgine Chiesa, Najma Douir, Gaëlle Mevel, and all the radiation therapists of the department for their help and the care they provide to patients.

viral shedding of $2019-\mathrm{nCoV}$ infections. medRxiv (2020) 2020.02.11.20021493. doi: 10.1101/2020.02.11.20021493

18. Ai T, Yang Z, Hou H, Zhan C, Chen C, Lv W, et al. Correlation of Chest CT and RT-PCR Testing in Coronavirus Disease 2019 (COVID-19) in China: A Report of 1014 Cases. Radiology (2020), 296(2):E32-40. doi: 10.1148/ radiol.2020200642

19. Fang Y, Zhang H, Xie J, Lin M, Ying L, Pang P, et al. Sensitivity of Chest CT for COVID-19: Comparison to RT-PCR. Radiology (2020) 296(2):E115-E117. doi: 10.1148/radiol.2020200432

20. Kim H, Hong H, Yoon SH. Diagnostic Performance of CT and Reverse Transcriptase-Polymerase Chain Reaction for Coronavirus Disease 2019: A Meta-Analysis. Radiology (2020) 296(3):E145-E155. doi: 10.1148/ radiol.2020201343

21. Hermans JJR, Groen Jw, Zwets E, Boxma-De Klerk BM, Van Werkhoven JM, Ong DSY, et al. Chest CT for triage during COVID-19 on the emergency department: myth or truth? Emerg Radiol (2020) 20:1-11. doi: 10.1007/ s10140-020-01821-1

22. Rubin GD, Ryerson CJ, Haramati LB, Sverzellati N, Kanne JP, Raoof S, et al. The Role of Chest Imaging in Patient Management during the COVID-19 Pandemic: A Multinational Consensus Statement from the Fleischner Society. Radiology (2020) 296(1):172-80. doi: 10.1148/radiol.2020201365

23. Peeling RW, Wedderburn CJ, Garcia PJ, Boeras D, Fongwen N, Nkengasong J, et al. Serology testing in the COVID-19 pandemic response. Lancet Infect Dis (2020) 20(9):e245-9. doi: 10.1016/S1473-3099(20)30517-X

24. To KK-W, Tsang OT-Y, Leung W-S, Tam AR, Wu T-C, Lung DC, et al. Temporal profiles of viral load in posterior oropharyngeal saliva samples and serum antibody responses during infection by SARS-CoV-2: an observational cohort study. Lancet Infect Dis (2020) 20(5):565-74. doi: 10.1016/S1473-3099(20)30196-1

25. Long Q-X, Liu B-Z, Deng H-J, Wu G-C, Deng K, Chen Y-K, et al. Antibody responses to SARS-CoV-2 in patients with COVID-19. Nat Med (2020) 26 (6):845-8. doi: 10.1038/s41591-020-0897-1

26. Zhao J, Yuan Q, Wang H, Liu W, Liao X, Su Y, et al. Antibody responses to SARS-CoV-2 in patients of novel coronavirus disease 2019. Clin Infect Dis (2020) ciaa344. doi: 10.1093/cid/ciaa344

27. Zhang B, Zhou X, Zhu C, Feng F, Qiu Y, Feng J, et al. Immune phenotyping based on neutrophil-to-lymphocyte ratio and IgG predicts disease severity and outcome for patients with COVID-19. medRxiv (2020) 7:157. doi: 10.1101/ 2020.03.12.20035048

28. World Health Organization Scientific Brief. Advice on the use of point-of-care immunodiagnostic tests for COVID-19. Available at: https://www.who.int/ news-room/commentaries/detail/advice-on-the-use-of-point-of-careimmunodiagnostic-tests-for-covid-19 [cite 22 juill 2020].

29. Minaya Flores P, Lasserre A, Zeghari-Squalli N, Carbonneil C, Morin-Surroca $\mathrm{M}$, Dalour S. Place des tests sérologiques rapides (TDR, TROD, autotests) dans la stratégie de prise en charge de la maladie COVID-19. Haute Autorité de santé (HAS) (2020). p. 34 . Available at: https://www.has-sante.fr/upload/docs/ application/pdf/2020-05/rapport_tests_serologiques_rapides_covid-19_vd.pdf.

30. Pereira GC, Traughber M, Muzic RF. The role of imaging in radiation therapy planning: past, present, and future. BioMed Res Int (2014) 2014:231090. doi: $10.1155 / 2014 / 231090$

31. Mettler FA, Huda W, Yoshizumi TT, Mahesh M. Effective Doses in Radiology and Diagnostic Nuclear Medicine: A Catalog. Radiology (2008) 248(1):25463. doi: 10.1148/radiol.2481071451

32. Smith-Bindman R, Moghadassi M, Wilson N, Nelson TR, Boone JM, Cagnon $\mathrm{CH}$, et al. Radiation Doses in Consecutive CT Examinations from Five University of California Medical Centers. Radiology (2015) 277(1):134-41. doi: 10.1148/radiol.2015142728

33. Benadjaoud MA, Bezin J, Veres A, Lefkopoulos D, Chavaudra J, Bridier A, et al. A multi-plane source model for out-of-field head scatter dose 
calculations in external beam photon therapy. Phys Med Biol (2012) 57 (22):7725-39. doi: 10.1088/0031-9155/57/22/7725

34. Caraiani C, Pop A, Calin A, Ciobanu L, Militaru C, Berghe A, et al. Incidental findings during follow-up scans in oncological patients. Clujul Med (2018) 91 (3):293-9. doi: 10.15386/cjmed-931

35. Wölfel R, Corman VM, Guggemos W, Seilmaier M, Zange S, Müller MA, et al. Virological assessment of hospitalized patients with COVID-2019. Nature (2020) 581(7809):465-9. doi: 10.1038/s41586-020-2196-x

36. He X, Lau EHY, Wu P, Deng X, Wang J, Hao X, et al. Temporal dynamics in viral shedding and transmissibility of COVID-19. Nat Med (2020) 26(5):6725. doi: 10.1038/s41591-020-0869-5

37. Ferretti L, Wymant C, Kendall M, Zhao L, Nurtay A, Abeler-Dörner L, et al. Quantifying SARS-CoV-2 transmission suggests epidemic control with digital contact tracing. Science (2020) 368(6491):eabb6936. doi: 10.1126/ science.abb6936

38. Backer JA, Klinkenberg D, Wallinga J. Incubation period of 2019 novel coronavirus (2019-nCoV) infections among travellers from Wuhan, China, 20-28 January 2020. Eurosurveillance (2020) 25(5):2000062. doi: 10.2807/ 1560-7917.ES.2020.25.5.2000062

39. Kuderer NM, Choueiri TK, Shah DP, Shyr Y, Rubinstein SM, Rivera DR, et al. Clinical impact of COVID-19 on patients with cancer (CCC19): a cohort study. Lancet (2020) 395(10241):1907-18. doi: 10.1016/S0140-6736(20) 31187-9

40. Salje H, Kiem CT, Lefrancq N, Courtejoie N, Bosetti P, Paireau J, et al. Estimating the burden of SARS-CoV-2 in France. Science (2020) 369 (6500):208-11. doi: 10.1126/science.abc3517
41. Bernheim A, Mei X, Huang M, Yang Y, Fayad ZA, Zhang N, et al. Chest CT Findings in Coronavirus Disease-19 (COVID-19): Relationship to Duration of Infection. Radiology (2020) 295(3):200463. doi: 10.1148/radiol. 2020200463

42. Lepelletier D, Grandbastien B, Romano-Bertrand S, Aho S, Chidiac C, Géhanno JF, et al. (2020). What face mask for what use in the context of COVID-19 pandemic? The French guidelines. J Hosp Infect 105(3):414-8. Advance online publication. doi: 10.1016/j.jhin.2020.04.036

43. Madariaga A, McMullen M, Sheikh S, Kumar R, Liu F-F, Zimmermann C, et al. COVID-19 testing in cancer patients: Does one size fit all? Clin Cancer Res (2020) 26(18):4737-42. doi: 10.1158/1078-0432.CCR-20-2224

Conflict of Interest: The authors declare that the research was conducted in the absence of any commercial or financial relationships that could be construed as a potential conflict of interest.

The handling editor is currently organizing a Research Topic with the authors SA and LD.

Copyright $\odot 2020$ Sun, Ammari, Bockel, Achkar, Merad, Dercle, Rivera, Chargari and Deutsch. This is an open-access article distributed under the terms of the Creative Commons Attribution License (CC BY). The use, distribution or reproduction in other forums is permitted, provided the original author(s) and the copyright owner(s) are credited and that the original publication in this journal is cited, in accordance with accepted academic practice. No use, distribution or reproduction is permitted which does not comply with these terms. 